\title{
Efficacy of a Community-Based Technology-Enabled Physical Activity Counseling Program for People With Knee Osteoarthritis: Proof-of-Concept Study
}

Linda C Li ${ }^{1,2}, \mathrm{PT}, \mathrm{PhD}$; Eric C Sayre ${ }^{2}, \mathrm{PhD}$; Hui Xie ${ }^{2,3}, \mathrm{PhD}$; Ryan S Falck ${ }^{1}, \mathrm{MSc}$ John R Best ${ }^{1}$, PhD; Teresa Liu-Ambrose $^{1}$, PT, PhD; Navi Grewal ${ }^{2}$; Alison M Hoens ${ }^{1}$, BSc (PT), MSc; Greg Noonan ${ }^{4}$, BSc (PT); Lynne M Feehan ${ }^{1,2}$, PT, PhD

\footnotetext{
${ }^{1}$ Department of Physical Therapy, University of British Columbia, Vancouver, BC, Canada

${ }^{2}$ Arthritis Research Canada, Richmond, BC, Canada

${ }^{3}$ Faculty of Health Sciences, Simon Fraser University, Burnaby, BC, Canada

${ }^{4}$ Mary Pack Arthritis Program, Vancouver General Hospital, Vancouver, BC, Canada
}

\section{Corresponding Author:}

Linda C Li, PT, PhD

Arthritis Research Canada

5591 No. 3 Road

Richmond, BC, V6X 2C7

Canada

Phone: 16042074020

Email: 1 li@ arthritisresearch.ca

\section{Abstract}

Background: Current practice guidelines emphasize the use of physical activity as the first-line treatment of knee osteoarthritis; however, up to $90 \%$ of people with osteoarthritis are inactive.

Objective: We aimed to assess the efficacy of a technology-enabled counseling intervention for improving physical activity in people with either a physician-confirmed diagnosis of knee osteoarthritis or having passed two validated criteria for early osteoarthritis.

Methods: We conducted a proof-of-concept randomized controlled trial. The immediate group received a brief education session by a physical therapist, a Fitbit Flex, and four biweekly phone calls for activity counseling. The delayed group received the same intervention 2 months later. Participants were assessed at baseline (T0) and at the end of 2 months (T1), 4 months (T2), and 6 months (T3). Outcomes included (1) mean time on moderate-to-vigorous physical activity (MVPA $\geq 3$ metabolic equivalents [METs], primary outcome), (2) mean time on MVPA $\geq 4$ METs, (3) mean daily steps, (4) mean time on sedentary activities, (5) Knee Injury and Osteoarthritis Outcome Score (KOOS), and (6) Partners in Health scale. Mixed-effects repeated measures analysis of variance was used to assess five planned contrasts of changes in outcome measures over measurement periods. The five contrasts were (1) immediate T1-T0 vs delayed T1-T0, (2) delayed T2-T1 vs delayed T1-T0, (3) mean of contrast 1 and contrast 2, (4) immediate T1-T0 vs delayed T2-T1, and (5) mean of immediate T2-T1 and delayed T3-T2. The first three contrasts estimate the between-group effects. The latter two contrasts estimate the effect of the 2-month intervention delay on outcomes.

Results: We recruited 61 participants (immediate: $n=30$; delayed: $n=31$ ). Both groups were similar in age (immediate: mean 61.3, SD 9.4 years; delayed: mean 62.1, SD 8.5 years) and body mass index (immediate: mean 29.2, SD $5.5 \mathrm{~kg} / \mathrm{m}^{2} ; \mathrm{delayed}$ : mean 29.2, SD $4.8 \mathrm{~kg} / \mathrm{m}^{2}$ ). Contrast analyses revealed significant between-group effects in MVPA $\geq 3$ METs (contrast 1 coefficient: 26.6, 95\% CI 4.0-49.1, $P=.02$; contrast 3 coefficient: 26.0, 95\% CI 3.1-49.0, $P=.03$ ), daily steps (contrast 1 coefficient: 1699.2 , 95\% CI 349.0-3049.4, $P=.02$; contrast 2 coefficient: $1601.8,95 \%$ CI 38.7-3164.9, $P=.045$; contrast 3 coefficient: 1650.5 , 95\% CI 332.3-2968.7; $P=.02$ ), KOOS activity of daily living subscale (contrast 1 coefficient: $6.9,95 \%$ CI $0.1-13.7, P=.047$; contrast 3 coefficient: $7.2,95 \%$ CI $0.8-13.6, P=.03$ ), and KOOS quality of life subscale (contrast 1 coefficient: 7.4, 95\% CI 0.0-14.7, $P=.049$; contrast 3 coefficient: 7.3 , 95\% CI $0.1-14.6, P=.048$ ). We found no significant effect in any outcome measures due to the 2-month delay of the intervention. 
Conclusions: Our counseling program improved MVPA $\geq 3$ METs, daily steps, activity of daily living, and quality of life in people with knee osteoarthritis. These findings are important because an active lifestyle is an important component of successful self-management.

Trial Registration: ClinicalTrials.gov NCT02315664; https://clinicaltrials.gov/ct2/show/NCT02315664 (Archived by WebCite at http://www.webcitation.org/6ynSgUyUC)

(J Med Internet Res 2018;20(4):e159) doi: 10.2196/jmir.8514

\section{KEYWORDS}

osteoarthritis; physical activity; wearables; goal setting; physiotherapy; eHealth

\section{Introduction}

Arthritis is the most common cause of severe chronic pain and disability worldwide. Analysis by the Arthritis Alliance of Canada estimates one new diagnosis of osteoarthritis (OA) every 60 seconds, resulting in nearly $30 \%$ of the employed labor force having difficulties working due to OA [1]. Current evidence supports the use of physical activity to manage OA due to its beneficial effects on pain, mobility, and quality of life [2,3]. It has been shown that moderate weight-bearing activities improve joint health by preserving glycosaminoglycan content in cartilage [4,5]. Furthermore, specific training that involves functional activities improves balance and proprioception, which in turn can contribute to improving pain and mobility [6,7]. The OA Research Society International recommends the use of physical activity and therapeutic exercise as a first-line treatment of knee OA [8]. Public health guidelines recommend more than 150 minutes a week of moderate-to-vigorous physical activity (MVPA) performed in bouts of 10 minutes or more [9]; however, a 2013 systematic review reported that only $13 \%$ of people with OA met this recommendation [10]. This concurs with another study using accelerometers that more than $90 \%$ of people with knee OA did not meet the physical activity guidelines [11]. The findings are particularly alarming because the evidence on the first-line treatment for OA has been consistent over a decade [12]. This represents a major knowledge-to-action gap.

Several modifiable risk factors are associated with low physical activity participation in people with arthritis. These include lack of motivation [13], doubts about the effectiveness of exercise [14], and lack of health professional advice [15]. Once patients start being active, they need feedback on their progress. A Cochrane review reported that "graded exercise activity," which initially focuses on simple activities and then gradually increases to more challenging ones, is effective for improving adherence in people with chronic musculoskeletal conditions [16]. Progression of activities and goals can be guided by a physical therapist (PT) [16].

We recently demonstrated feasibility of a physical activity counseling program with the use of a Fitbit wrist band in 34 people with knee OA [17]. Compared to controls, those who received the program showed a trend of increased MVPA and perceived self-management capacity after 1 month [17]. The findings supported further research on this program. The purpose of the current study was to assess the efficacy of the program for improving physical activity participation, disease status, and perceived self-management capacity in people with knee OA.

\section{Methods}

\section{Study Design and Participant Eligibility}

Monitor-OA was a proof-of-concept study that used a randomized, delayed-control design, whereby the randomization determined the timing of when the intervention was provided (ie, immediately vs a 2-month delay). As such, efficacy was assessed within a conventional randomized controlled trial (RCT), with an intervention group and a control group, at 2 months, whereas all participants received the intervention beyond this time. This study design is best suited for interventions that include components that are likely beneficial and low risk to participants, such as physical activity counseling.

Eligible individuals were those who had a physician-confirmed diagnosis of knee OA or passed two criteria for early OA: (1) aged 50 years and (2) had experienced pain or discomfort in or around the knee during the previous year lasting more than 28 separate or consecutive days. In a community-based study [18], 191 of 195 (97\%) urban-dwelling participants who met these criteria also met the American College of Rheumatology clinical criteria for knee OA [19].

We excluded individuals who:

1. had a diagnosis of inflammatory arthritis, connective tissue diseases, fibromyalgia, or gout;

2. had used disease-modifying antirheumatic drugs or gout medications;

3. had knee arthroplasty;

4. were on a waitlist to receive knee or hip arthroplasty;

5. had any surgery in the back, hip, knee, foot, or ankle joint in the past 12 months;

6. had acute knee injury in the past 6 months;

7. had received a steroid injection or hyaluronate injection in a knee in the last 6 months;

8. had a body mass index (BMI) of $40 \mathrm{~kg} / \mathrm{m}^{2}$ or higher;

9. did not have an email address or daily access to a personal computer with Internet access;

10. were unable to attend the required education session in person;

11. were using medications that impaired activity tolerance (eg, beta-blockers); and

12. had an inappropriate level of risk for increasing their unsupervised physical activity.

Potential participants completed the Physical Activity Readiness Questionnaire (PAR-Q [20]; 2014 version). If a potential risk was identified by the PAR-Q, physician confirmation in writing 
was required to ensure that the person was able to be physically active without supervision of a health professional.

Participants were recruited from the Mary Pack Arthritis Program in Vancouver, BC, Canada. Study information was also posted on social media (Facebook, Twitter, Kijiji, and Craigslist) and the Arthritis Research Canada website. In addition, emails about the study were sent by the Arthritis Consumer Experts, a nonprofit patient education organization, to their members. After completing the baseline assessment, eligible participants were randomly assigned to the immediate group or the delayed group (ie, control) in 1:1 allocation ratio. The delayed group received the same intervention as the immediate group after a 2-month wait. We performed randomization using computer-generated random numbers in variable block sizes.

\section{Intervention}

The intervention involved participants attending a 1.5-hour session, where they received (1) 15-minute standardized education about physical activity, (2) a Fitbit Flex, and (3) individual counseling with a study PT who was trained in motivational interviewing [21]. The choice of a face-to-face session over the use of videoconferencing technology was to maximize the opportunity for participants and PTs to established rapport [22,23]. The education portion, delivered in groups of two to four participants, addressed the benefits of an active lifestyle, the detrimental effect of sedentary behavior, and ways to be active without aggravating OA symptoms. The individual counseling portion followed the Brief Action Planning approach [24], whereby PTs guided participants to identify activity goals, develop an action plan, and identify barriers and solutions. The PTs used the SMART (specific, measurable, attainable, relevant, time-bound) principle during goal setting (eg, 30 minutes of brisk walking in the neighborhood in the evening three times a week). Participants were then asked to rate their confidence in executing the plan on a zero to 10 scale, with 10 meaning very confident. The process was repeated until the confidence rating reached 7 or higher out of 10. For sedentary behaviors, the PTs began by asking participants to estimate their sitting time in a normal day and identify ways to break up the sitting time. They then repeated the goal setting and confidence assessment.

Participants then received a Fitbit Flex to be worn at the wrist of the nondominant side 24 hours a day except during water-based activity or when charging. The physical activity data were wirelessly synchronized with Fitbit's online Dashboard that could be viewed only by the participants and their study PTs. During the intervention period, the PT reviewed the participant's physical activity on the Dashboard and progressively modified their SMART goals during four biweekly 20-minute phone calls. Participants could also contact the PT via email in-between the scheduled calls. At the end of the intervention, participants could keep the Fitbit.

Four PTs with a primary caseload consisting of patients with arthritis were trained to deliver the education and counseling components. Three of them were from the public sector (Vancouver Coastal Health Authority, Vancouver, BC, Canada), and one worked in a mix of public and private practices. One PT also participated in our previous feasibility study [17] and provided feedback to refine the intervention for the current project. The PTs attended a 2-day introductory motivational interview course offered by the University of British Columbia Extended Learning program. Before data collection, we held two orientation sessions ( 2 hours each) for the PTs to review the study protocol and practice the counseling component.

\section{Outcome Measures}

Participants were assessed at baseline (T0) and the end of 2 months (T1), 4 months (T2), and 6 months (T3). Our primary outcome measure was mean daily time performing MVPA at $\geq 3$ metabolic equivalents, or METs (MVPA $\geq 3$ METs; performed in both ambulatory and nonambulatory activities throughout the day) measured with SenseWear Mini, a multisensor monitor that was worn on the upper arm over the triceps. SenseWear integrates triaxial accelerometer data, physiological sensor data, and personal demographic information to provide estimates of steps and energy expenditure. Tierney et al [25] showed that SenseWear was a valid tool for estimating energy expenditure during activities of daily living in people with arthritis (intraclass correlation coefficient $[\mathrm{ICC}]=0.72$ ). A strong relationship was also found between SenseWear and indirect calorimetry measures of energy expenditure for activities of daily living (Pearson $r=.85$ ) [25]. SenseWear can be worn 24 hours a day; hence, it can capture a full picture of physical activity and the off-body time throughout the day $[26,27]$. Participants wore a SenseWear for 7 days at each assessment. Almeida et al [28] determined that a minimum of 4 days of wear was required to reliably assess energy expenditure from different levels of physical activity in people with arthritis (ICC >0.80).

We performed additional analysis with a MVPA cut-off at $\geq 4$ METs, which reflected an activity level of brisk walking and higher (ie, purposeful ambulatory activities) [29]. Other secondary outcomes included the daily mean time spent in sedentary activities [30], the Knee Injury and OA Outcome Score (KOOS) [31,32], and the Partners in Health scale [33]. An important feature of SenseWear is its ability to differentiate between sedentary and light activities [34], making it an ideal instrument to assess sedentary activities. For sedentary activities, we calculated the mean daily time spent with an energy expenditure of $\leq 1.5 \mathrm{METs}$, occurring in bouts of 20 minutes or more during waking hours [35-38].

The KOOS consists of five subscales: knee pain, stiffness, activity of daily living, sports/recreation, and quality of life. It was originally developed for people recovering from anterior cruciate ligament and meniscus injury and has been validated in people with OA [31,32]. The Partners in Health scale is a 12-item measure designed to assess perceived self-management capacity via subjective knowledge of the health condition and treatment, and perceived self-management behaviors (eg, adopting a healthy lifestyle; Cronbach alpha=.82) [33]. We also tracked self-reported adverse events (falls, cardiovascular and musculoskeletal events) [39] using a monthly log.

\section{Sample Size Justification and Data Analysis}

Our recruitment strategy enabled the study to enroll at least 60 eligible participants over 12 months. For a proof-of-concept 
study, it is reasonable to expect a moderately large difference between groups after the intervention. Based on our feasibility study, we estimated the standard deviation of the change in MVPA $\geq 3$ METs (primary outcome measure) from T0-T1 to be 40 minutes. This resulted in $81.5 \%$ power to detect a 30 -minute difference between groups in the T1-T0 change via a two-sided test at alpha level of .05.

Descriptive analysis was used to summarize participant characteristics, comorbid conditions, and adverse events. We generated plots that included means and standard errors for the outcome measures at each time point for both groups for all outcome measures.

An intention-to-treat analysis was performed by a biostatistician who was blinded to the group assignment. Quantile-quantile plots were used to assess normality of the outcome variables. Mixed-effects repeated measures analysis of variance was used to assess five planned contrasts of changes in outcome measures over measurement periods. They were:

1. Contrast 1: immediate group T1-T0 vs delayed group T1-T0;

2. Contrast 2: delayed group T2-T1 vs delayed group T1-T0;

3. Contrast 3: mean of contrast 1 and contrast 2;

4. Contrast 4: immediate group T1-T0 vs delayed group $\mathrm{T} 2-\mathrm{T} 1$; and

5. Contrast 5: mean of immediate group T2-T1 and delayed group T3-T2.

The first three contrasts estimate the effect of the intervention versus control. The latter two contrasts estimate the effect of the delay on the intervention. Contrast 1 is the between-group contrast estimate for the 2-month effect of the program, similar to analysis from a parallel design. Contrast 2 is the within-group contrast estimate for the 2-month effect of the program. One benefit of the delayed-control design is that experimental units can serve as their own control to improve efficiency and precision of treatment effect estimation. To further improve the treatment effect estimation efficiency, we combined contrast 1 and 2 by taking their mean (contrast 3 ). Contrast 4 examines the 2-month effect of the program when it was delayed for 2 months. Contrast 5 combines data from both groups to assess the lastingness of the effect, when the program ended 2 months before assessment. No adjustment was made for multiple comparisons because type II error is a greater concern than type I error in proof-of-concept studies [40,41].

\section{Ethics Approval}

The research protocol was approved by the University of British Columbia Behavioral Research Ethics Board (application number: H14-01762) and was published in ClinicalTrials.gov (NCT02315664).

\section{Results}

In 2015-2016, 278 people indicated an interest to participate and 64 met the eligibility criteria (Figure 1). Of those, we recruited 61 participants (immediate group: $n=30,73 \%, 22 / 30$ women; delayed group: $\mathrm{n}=31,90 \%, 28 / 31$ women). Both groups were similar in age (immediate group: mean 61.3, SD 9.4 years; delayed group: mean 62.1, SD 8.5 years) and BMI (immediate group: mean 29.2 , SD $5.5 \mathrm{~kg} / \mathrm{m}^{2}$; delayed group: mean 29.2 , SD $4.8 \mathrm{~kg} / \mathrm{m}^{2}$; Table 1).

Multimedia Appendix 1 and Figures 2-11 present the results of outcome measures from four time points. Prespecified contrast analyses revealed significant effects as follows: mean time on MVPA $\geq 3$ METs (contrast 1 coefficient: 26.6, 95\% CI 4.0-49.1, $P=.02$; contrast 3 coefficient: $26.0,95 \%$ CI 3.1-49.0, $P=.03$ ), mean daily steps (contrast 1 coefficient: 1699.2, 95\% CI 349.0-3049.4, $P=.02$; contrast 2 coefficient: $1601.8,95 \%$ CI 38.7-3164.9, $P=.045$; contrast 3 coefficient: $1650.5,95 \% \mathrm{CI}$ $332.3-2968.7, P=.02)$, KOOS activity of daily living subscale (contrast 1 coefficient: $6.9,95 \%$ CI $0.1-13.7, P=.047$; contrast 3 coefficient: $7.2,95 \%$ CI $0.8-13.6, P=.03)$, KOOS quality of life subscale (contrast 1 coefficient: $7.4,95 \%$ CI $0.0-14.7$, $P=.049$; contrast 3 coefficient: 7.3 , 95\% CI 0.1-14.6, $P=.048$ ). We found no significant effect in any outcome measures in the other contrast analyses. No adverse event associated with the intervention (eg, falls, cardiovascular and musculoskeletal events) was reported by participants during the study. 
Figure 1. Consolidated Standards of Reporting Trials (CONSORT) flowchart.

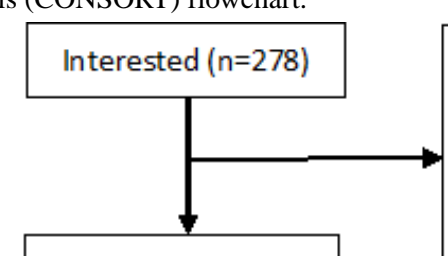

Assessed for eligibility $(n=217)$

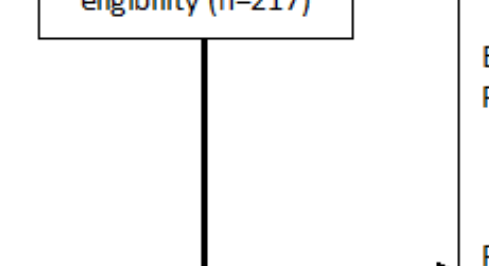

\section{$\longrightarrow$}

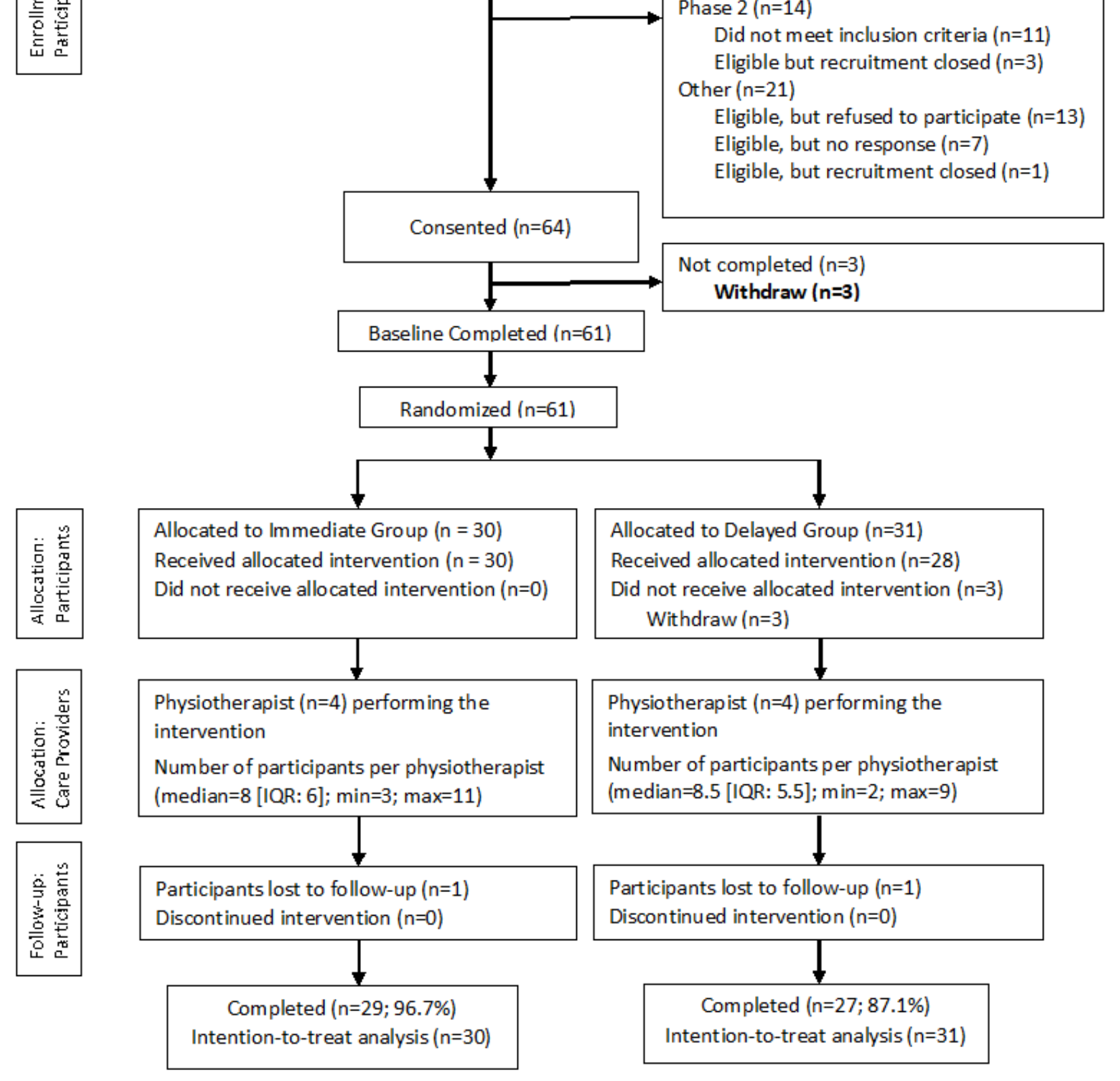

Not assessed for eligibility $(n=61)$

Participant contacted, but no answer $(\mathrm{n}=41)$ Incorrect email address $(n=3)$ Refused to participate $(n=16)$ Language barrier $(n=1)$

\section{Excluded $(n=153)$}

Phase $1(n=118)$

Did not meet inclusion criteria $(n=112)$

Eligible, but refused to participate $(n=4)$

Eligible, but no response $(n=2)$

Phase $2(n=14)$

Did not meet inclusion criteria $(n=11)$

Eligible but recruitment closed $(n=3)$

Other $(n=21)$

Eligible, but refused to participate $(n=13)$

Eligible, but no response ( $n=7$ )

Eligible, but recruitment closed $(n=1)$

Not completed $(n=3)$

Withdraw $(n=3)$ 
Table 1. Baseline characteristics of immediate group and delayed group participants.

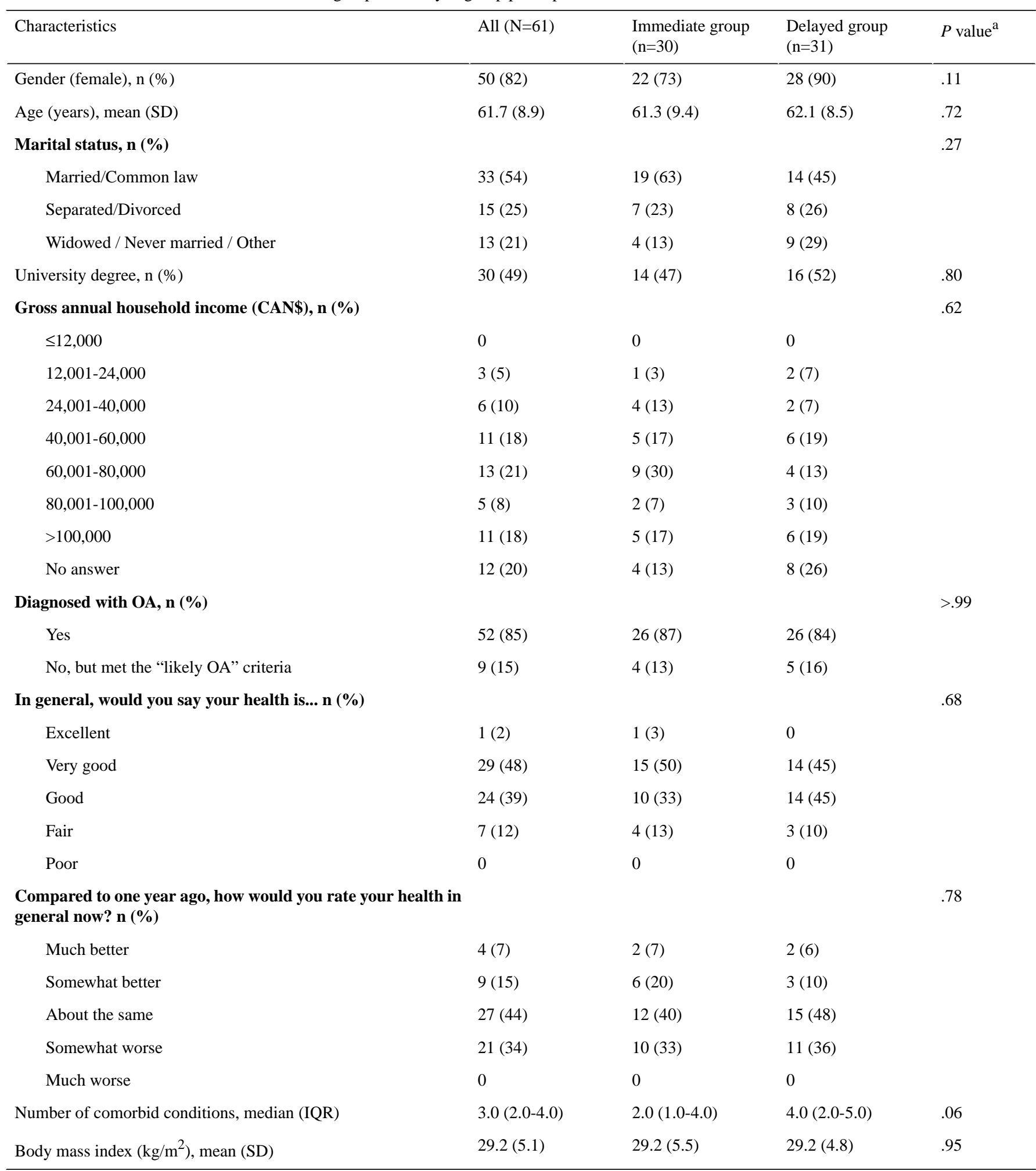

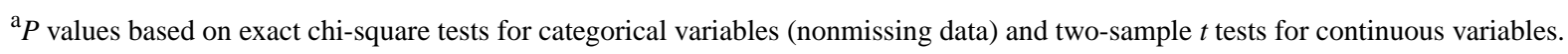


Figure 2. Bouted moderate to vigorous physical activity ( $\geq 3$ metabolic equivalent tasks [METs]).

\section{A}

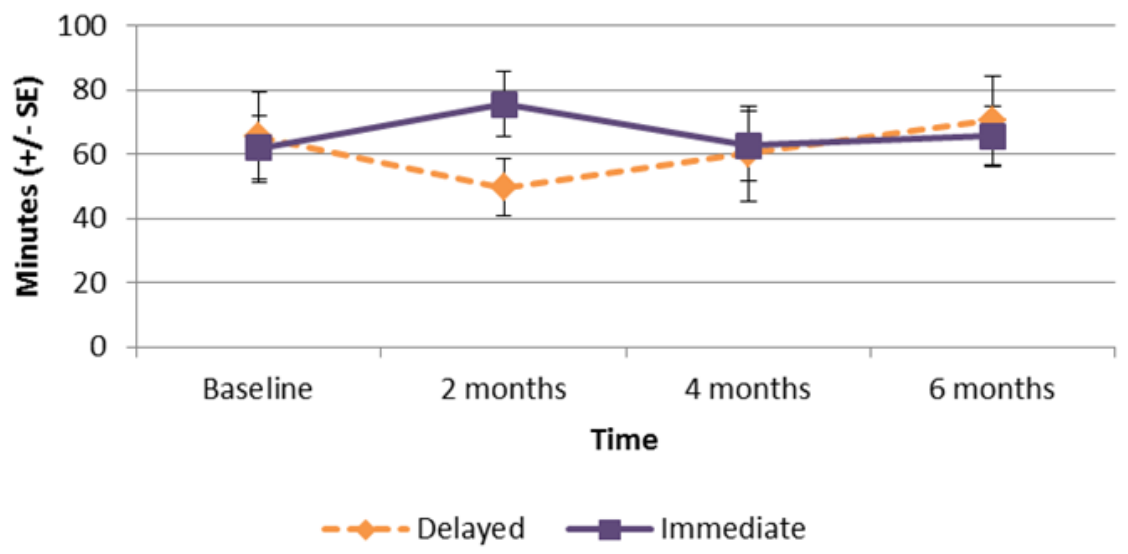

Figure 3. Bouted moderate to vigorous physical activity ( $\geq 4$ METs).

B

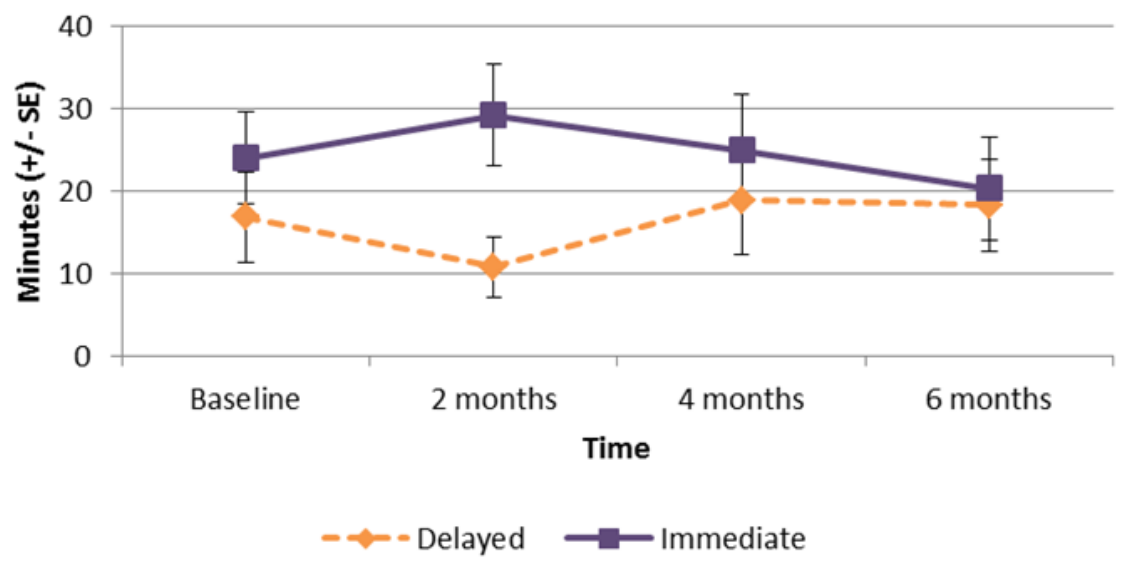

Figure 4. Bouted sedentary time.

C

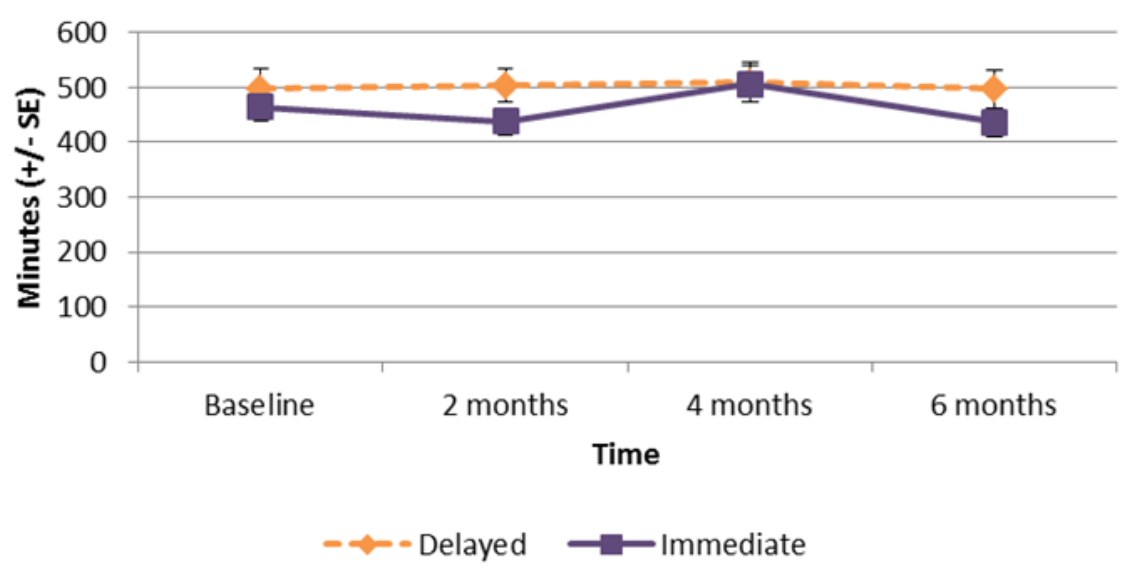


Figure 5. Mean daily step count.

D

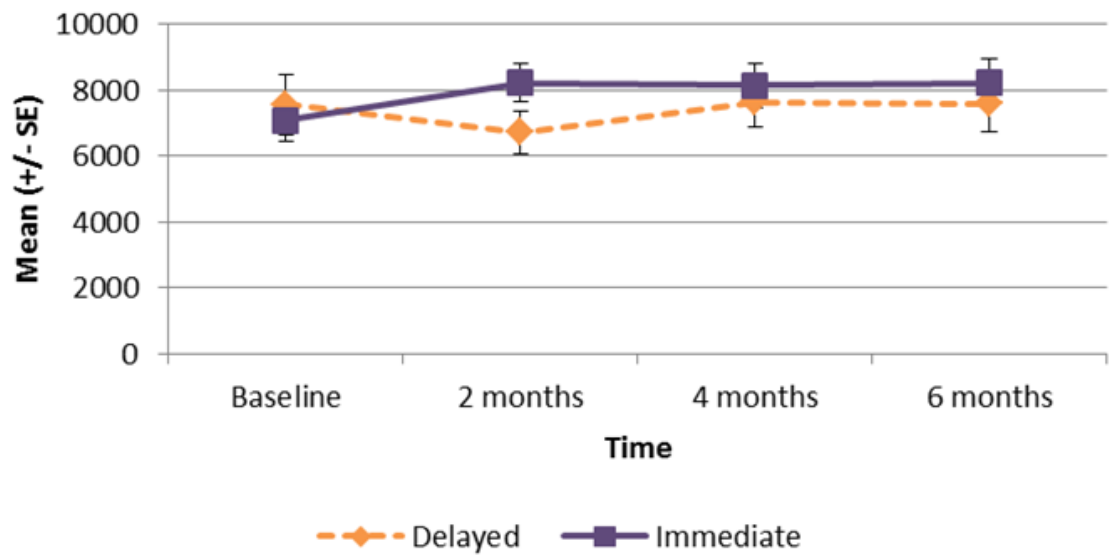

Figure 6. Knee Injury and Osteoarthritis Outcome Score (KOOS) symptoms subscale.

E

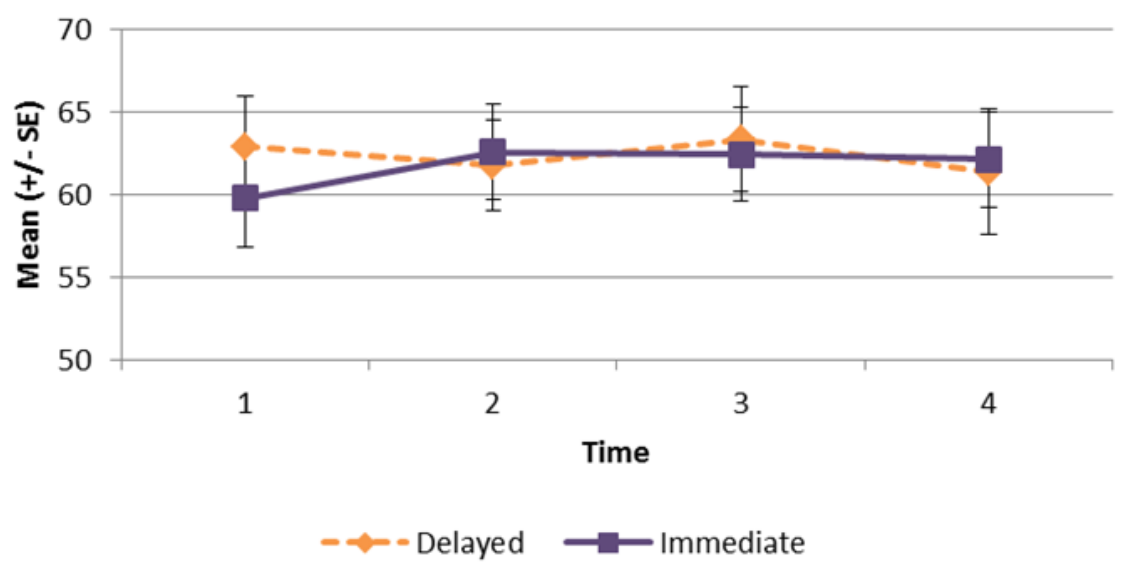

Figure 7. KOOS pain subscale.

$\mathbf{F}$

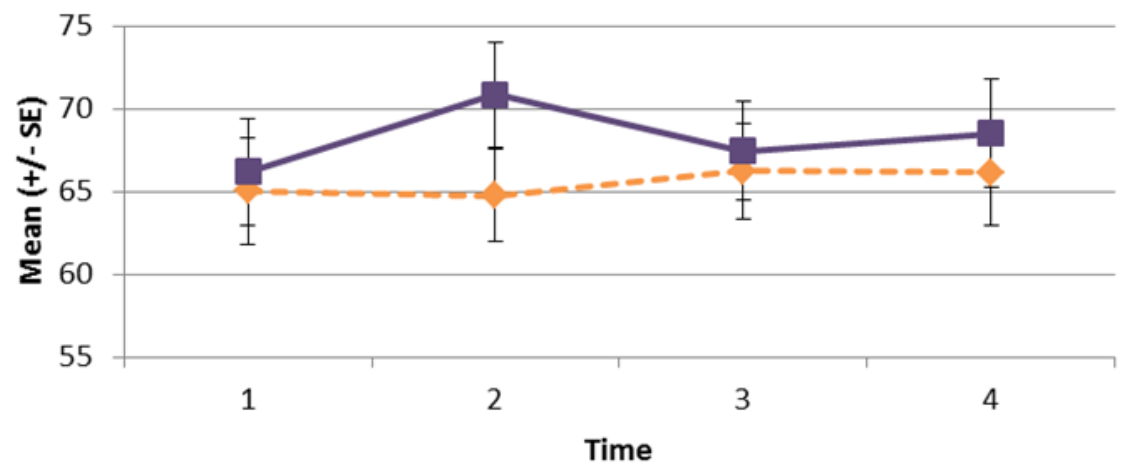

$-\diamond-$ Delayed $\longrightarrow$-Immediate 
Figure 8. KOOS sports and recreation subscale.

$\mathbf{G}$

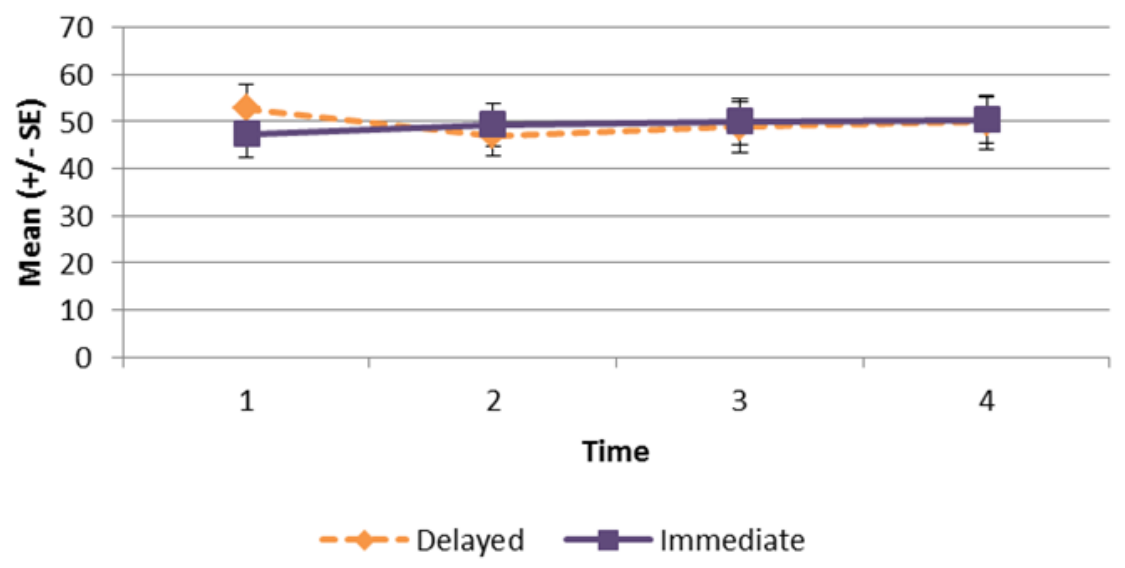

Figure 9. Partners in Health scale.

H
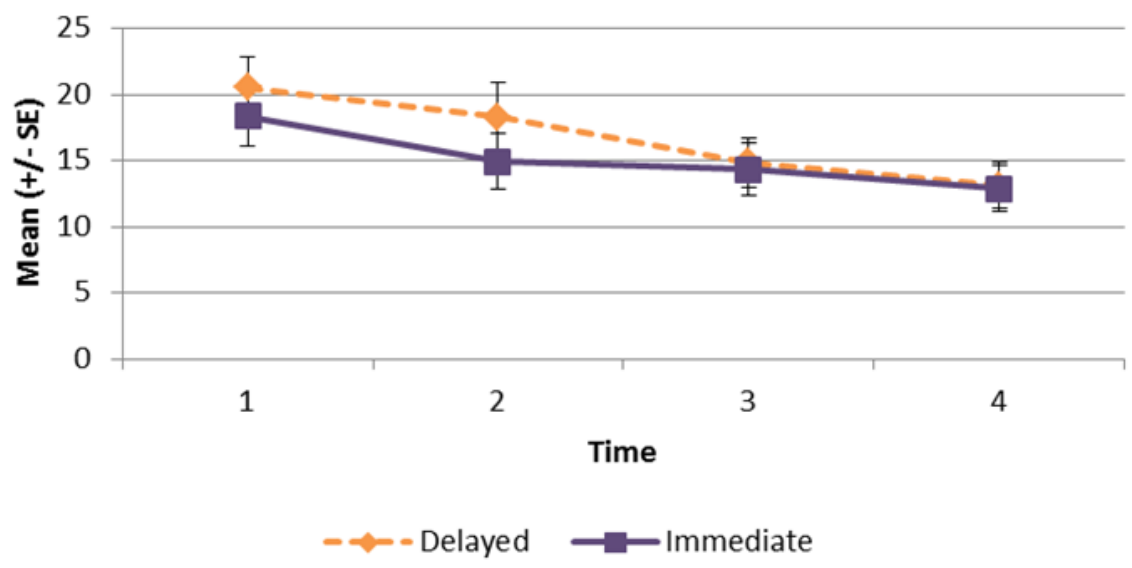

Figure 10. KOOS activities of daily living subscale.

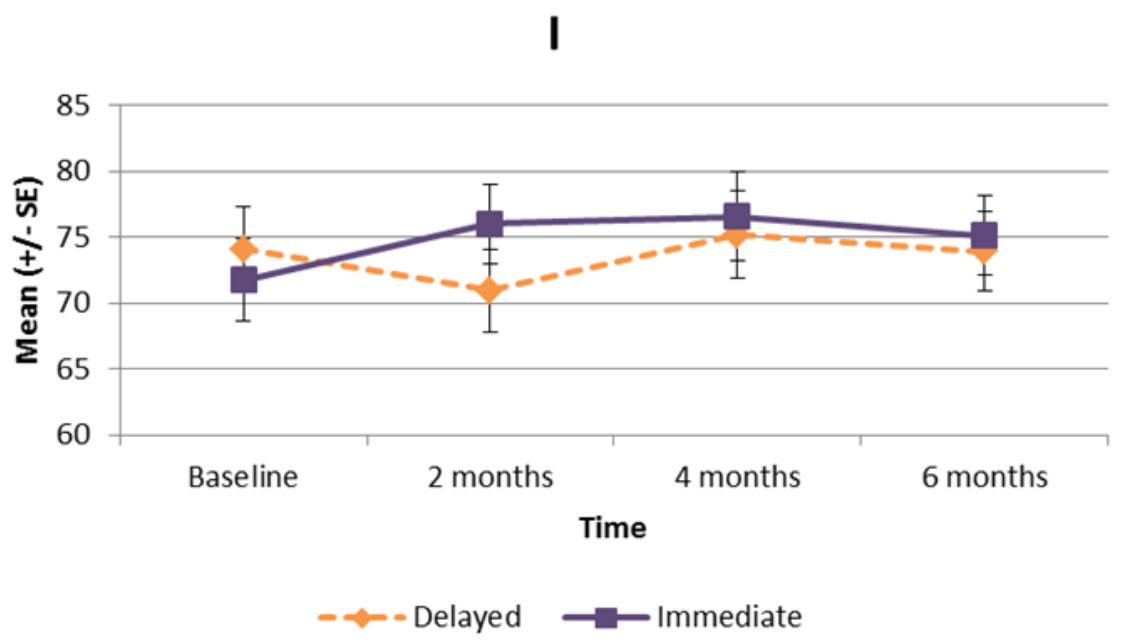


Figure 11. KOOS quality of life subscale.

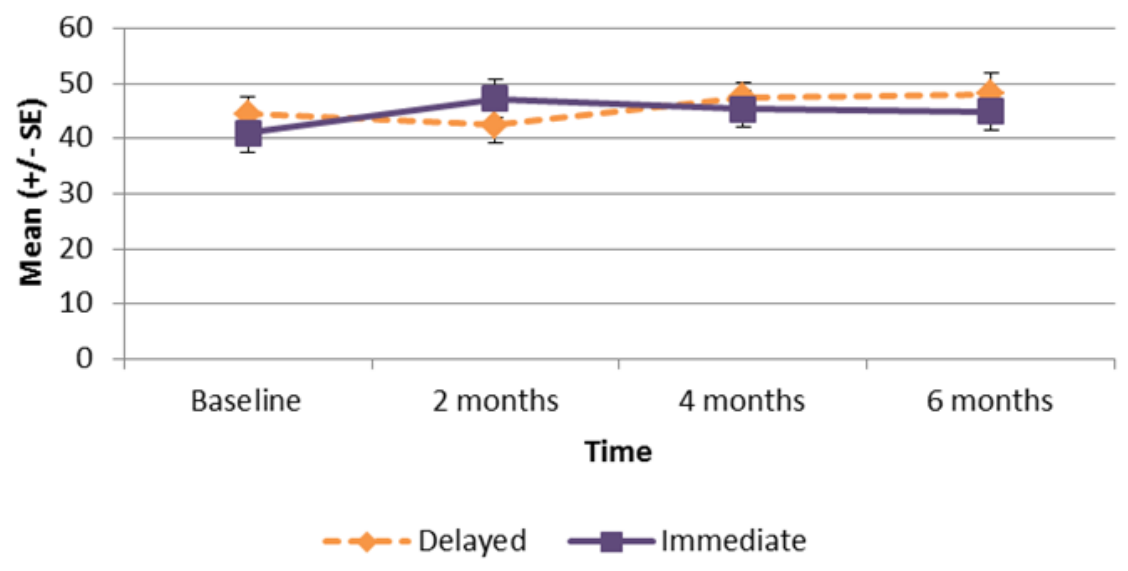

\section{Discussion}

This proof-of-concept study showed that our intervention had a significant effect on time spent on physical activity (MVPA $\geq 3$ METs) by people with knee OA compared to controls. The program also had a positive effect on step count, as well as activity of daily living and quality of life measured by the KOOS. Furthermore, the 2-month delay did not affect how participants responded to the program. This is noteworthy for the design of future RCTs in which the use of a "delay control" is considered. Our findings match those of previous studies that found individualized programs and self-management techniques could enhance physical activity adherence among people with chronic musculoskeletal conditions [16,17]. However, the findings should be viewed in the context that compared to the general population with knee OA, our participants were more active (baseline MVPA $\geq 3$ METs for more than 60 minutes per day). A 2011 study in the United States using accelerometers found that more than $90 \%$ of people with knee OA failed to meet the physical activity guidelines of 150 minutes of MVPA per week [11].

Our positive results may be explained by two reasons. First, we employed a variety of behavior change techniques to promote physical activity. In a review of 13 consumer wearables, Lyons et al [42] found that Fitbit used behavior change techniques such as goal setting, feedback, self-monitoring, social support, social comparison, as well as providing instructions and rewards. Although many of these techniques are in line with recommendations from social cognitive theory [43], techniques such as action planning, problem solving, and behavioral practice/rehearsal are absent with the use of wearables alone [42]. In a 2015 systematic review, Lewis et al [44] reported that wearable-only interventions tend to produce only modest effects on improving physical activity behavior. Therefore, we included PT counselors with expertise in arthritis care and motivational interview skills to prompt participants to plan and practice their activities, and to identify potential barriers and solutions during the follow-up calls. Our findings also echo those of a recent RCT on a 12-week intervention involving an Internet-based physical activity program with the use of an accelerometer and remote coaching. Broekhuizen et al [45] reported that the intervention improved the emotional and mental health among community-dwelling older adults (mean age 65 years).

The second reason was related to the experience and training of our study PTs. Previous studies in health counseling have stressed the importance of the experience and skill of counselors in behavioral interventions [46,47]. In this study, all PTs had a clinical caseload primarily in arthritis, received the essential training on motivational interviewing, and were familiar with the counseling protocol. Taken together, these reasons might have contributed to the positive effects of the intervention.

However, we did not observe a significant effect in the mean time spent in sedentary activities. From the feasibility study, we learned that increasing physical activity and reducing sedentary behavior (eg, prolonged sitting) required distinct counseling approaches, and that practice would be required by the PTs to deliver the intervention [17]. Hence, all study PTs were provided opportunities to practice the counseling procedure with the investigators before data collection. We did not make major modification to the counseling protocol, and it was possible that some challenges persisted for participants to identify ways to break up their sitting time. It should be noted that sedentary behavior is linked to individuals' habitual routines [48-51]. Although the general recommendation of MVPA can be accomplished in short daily episodes (eg, 30 minutes of brisk walking), reducing sedentary time requires people adjusting their habits throughout the day (eg, computer/mobile device use, television viewing). Therefore, different counseling strategies are likely required for the two behaviors.

In light of the findings, we suggest two modifications for the physical activity counseling program. First, the counseling conversation should begin by examining the individual's habitual routines during a typical workday and a non-work/weekend day, and then focus on periods when prolonged sitting occurs. This would allow the conversation to center on identifying opportunities, challenges, and solutions to break up sitting time when it is feasible for the person. Second, a more flexible system would be needed for setting personal goals to reduce sedentary time. The current Fitbit-manufactured apps, both the Web-based and mobile versions, reward users if they take 250 steps or more 
(approximately 2-3 minutes of walking) in a given hour. Although the parameter meets the general recommendation of standing up and moving after 30 minutes of uninterrupted sitting, it does not allow the flexibility to individualize goals to break up sitting. We suggest that future Fitbit-compatible apps should include functions for users to set personalized goals on the frequency and duration to break up their sitting time during the day, and to receive feedback on their goal attainment. This would provide users with positive reinforcement to adopt the current recommendation of reducing extended periods of sitting.

This study has a few limitations. With the use of a delayed-control design in which the participants in the control arm received treatment after a 2-month delay, efficacy of the physical activity counseling intervention could only be assessed at 2 months. Hence, the long-term effect of the intervention remained unclear. Furthermore, our sample was relatively active; hence, the results may not be generalizable to people with knee OA who are more sedentary. The results also may not be generalizable to men because $82 \%$ of the participants were women. Finally, we have identified several shortcomings in the counseling program that may have limited its potential to affect the behavioral and health-related outcomes. Despite these limitations, this proof-of-concept study has demonstrated a significant effect of a multifaceted counseling intervention on improving physical activity participation. We have since applied these learnings to improve the next iteration of the program. Specifically, it now includes a new sedentary counseling strategy and a Fitbit-compatible Web app with enhanced functionality for setting goals and rewarding behaviors that break up prolonged sitting [52]. The modified program is currently being tested in a RCT involving people with rheumatoid arthritis and systematic lupus erythematosus (ClinicalTrial.gov identifier: NCT02554474) [53].

\section{Acknowledgments}

The authors are grateful for the collaboration of patient partner Cheryl Koehn (Arthritis Consumer Experts) and physical therapists Karen Tsui, Valerie Gray, Carla Wiebe, and Wendy Watson. This study was supported by a Vancouver Coastal Health Research Institute Innovation \& Translational Research Award.

LCL and TL-A are supported by the Canada Research Chair Program and the Michael Smith Foundation for Health Research (MSFHR) Scholar Award. LCL is also supported by the Harold Robinson/Arthritis Society Chair in Arthritic Diseases. HX is supported by the Maureen and Milan Ilich / Merck Chair in Statistics for Arthritis and Musculoskeletal Diseases. RSF is funded by the University of British Columbia Rehabilitation Sciences Scholarship and the Omer H Patrick II Memorial Award.

\section{Conflicts of Interest}

None declared.

\section{Multimedia Appendix 1}

Participant outcomes and results of contrast analyses.

\section{[PDF File (Adobe PDF File), 54KB-Multimedia Appendix 1]}

\section{References}

1. Bombardier C, Hawker G, Mosher D. The Impact of Arthritis in Canada: Today and Over the Next 30 Years. Toronto, ON: Arthritis Alliance of Canada; 2011.

2. Brosseau L, MacLeay L, Robinson V, Wells G, Tugwell P. Intensity of exercise for the treatment of osteoarthritis. Cochrane Database Syst Rev 2003(2):CD004259. [doi: 10.1002/14651858.CD004259] [Medline: 12804510$]$

3. Zhang W, Moskowitz RW, Nuki G, Abramson S, Altman RD, Arden N, et al. OARSI recommendations for the management of hip and knee osteoarthritis, part I: critical appraisal of existing treatment guidelines and systematic review of current research evidence. Osteoarthritis Cartilage 2007 Sep;15(9):981-1000 [FREE Full text] [doi: 10.1016/j.joca.2007.06.014] [Medline: 17719803 ]

4. Roos E, Dahlberg L. Positive effects of moderate exercise on glycosaminoglycan content in knee cartilage: a four-month, randomized, controlled trial in patients at risk of osteoarthritis. Arthritis Rheum 2005 Nov;52(11):3507-3514 [FREE Full text] [doi: 10.1002/art.21415] [Medline: 16258919]

5. Munukka M, Waller B, Häkkinen A, Nieminen M, Lammentausta E, Kujala U, et al. Physical activity Is related with cartilage quality in women with knee osteoarthritis. Med Sci Sports Exerc 2017 Jul;49(7):1323-1330. [doi: 10.1249/MSS.0000000000001238] [Medline: 28240703]

6. Skou S. Roos EM: Good Life with osteoArthritis in Denmark (GLA:D)vidence-based education and supervised neuromuscular exercise delivered by certified physiotherapists nationwide. BMC Musculoskelet Disord 2017;18(1):72. [doi: 10.1186/s12891-017-1439-y] [Medline: 5297181]

7. Davis AM, Kennedy D, Wong R, Robarts S, Skou S, McGlasson R, et al. Cross-cultural adaptation and implementation of Good Life with osteoarthritis in Denmark (GLA:D $\left.{ }^{\mathrm{TM}}\right)$ : group education and exercise for hip and knee osteoarthritis is feasible in Canada. Osteoarthritis Cartilage 2018 Feb;26(2):211-219. [doi: 10.1016/j.joca.2017.11.005] [Medline: 29146385] 
8. Zhang W, Moskowitz RW, Nuki G, Abramson S, Altman RD, Arden N, et al. OARSI recommendations for the management of hip and knee osteoarthritis, Part II: OARSI evidence-based, expert consensus guidelines. Osteoarthritis Cartilage 2008 Feb;16(2):137-162 [FREE Full text] [doi: 10.1016/j.joca.2007.12.013] [Medline: 18279766]

9. Haskell W, Lee I, Pate R, Powell K, Blair S, Franklin B, et al. Physical activity and public health: updated recommendation for adults from the American College of Sports Medicine and the American Heart Association. Med Sci Sports Exerc 2007 Aug;39(8):1423-1434. [doi: 10.1249/mss.0b013e3180616b27] [Medline: 17762377]

10. Wallis JA, Webster KE, Levinger P, Taylor NF. What proportion of people with hip and knee osteoarthritis meet physical activity guidelines? A systematic review and meta-analysis. Osteoarthritis Cartilage 2013 Nov;21(11):1648-1659 [FREE Full text] [doi: 10.1016/j.joca.2013.08.003] [Medline: 23948979]

11. Dunlop DD, Song J, Semanik PA, Chang RW, Sharma L, Bathon JM, et al. Objective physical activity measurement in the osteoarthritis initiative: are guidelines being met? Arthritis Rheum 2011 Nov;63(11):3372-3382 [FREE Full text] [doi:

10.1002/art.30562] [Medline: 21792835]

12. Nelson AE, Allen KD, Golightly YM, Goode AP, Jordan JM. A systematic review of recommendations and guidelines for the management of osteoarthritis: The chronic osteoarthritis management initiative of the U.S. bone and joint initiative. Semin Arthritis Rheum 2014 Jun;43(6):701-712. [doi: 10.1016/j.semarthrit.2013.11.012] [Medline: 24387819]

13. Gyurcsik N, Brawley L, Spink K, Brittain D, Fuller D, Chad K. Physical activity in women with arthritis: examining perceived barriers and self-regulatory efficacy to cope. Arthritis Rheum 2009 Aug 15;61(8):1087-1094 [FREE Full text] [doi: 10.1002/art.24697] [Medline: 19644901]

14. Der Ananian C, Wilcox S, Saunders R, Watkins K, Evans A. Factors that influence exercise among adults with arthritis in three activity levels. Prev Chronic Dis 2006 Jul;3(3):A81 [FREE Full text] [Medline: 16776882]

15. Henchoz Y, Zufferey P, So A. Stages of change, barriers, benefits, and preferences for exercise in RA patients: a cross-sectional study. Scand J Rheumatol 2013;42(2):136-145. [doi: 10.3109/03009742.2012.724707] [Medline: 23244196]

16. Jordan J, Holden M, Mason EJ, Foster NE. Interventions to improve adherence to exercise for chronic musculoskeletal pain in adults. Cochrane Database Syst Rev 2010 Jan 20(1):CD005956. [doi: 10.1002/14651858.CD005956.pub2] [Medline: 20091582]

17. Li L, Sayre E, Xie H, Clayton C, Feehan LM. A community-based physical activity counselling program for people with knee osteoarthritis: feasibility and preliminary efficacy of the track-OA study. JMIR Mhealth Uhealth 2017 Jun 26;5(6):e86 [FREE Full text] [doi: 10.2196/mhealth.7863] [Medline: 28652228]

18. Marra C, Cibere J, Tsuyuki R, Soon J, Esdaile J, Gastonguay L, et al. Improving osteoarthritis detection in the community: pharmacist identification of new, diagnostically confirmed osteoarthritis. Arthritis Rheum 2007 Oct 15;57(7):1238-1244 [FREE Full text] [doi: 10.1002/art.23019] [Medline: 17907209]

19. Altman R, Asch E, Bloch D, Bole G, Borenstein D, Brandt K, et al. Development of criteria for the classification and reporting of osteoarthritis. Classification of osteoarthritis of the knee. Diagnostic and Therapeutic Criteria Committee of the American Rheumatism Association. Arthritis Rheum 1986 Aug;29(8):1039-1049. [Medline: 3741515]

20. Thomas S, Reading J, Shephard RJ. Revision of the Physical Activity Readiness Questionnaire (PAR-Q). Can J Sport Sci 1992 Dec;17(4):338-345. [Medline: 1330274$]$

21. Rollnick S, Miller W, Butler CC. Motivational Interviewing in Health Care: Helping Patients Change Behavior. New York: Guilford Press; Jul 02, 2009.

22. Miller W, Rollnick S. Motivational Interviewing: Helping People Change. 3rd ed. New York: Guilford Press; 2012.

23. Richards J, Hillsdon M, Thorogood M, Foster C. Face-to-face interventions for promoting physical activity. Cochrane Database Syst Rev 2013 Sep 30(9):CD010392. [doi: 10.1002/14651858.CD010392.pub2] [Medline: 24085592]

24. Gutnick D, Reims K, Davis C, Gainforth H, Jay M, Cole S. Brief action planning to facilitate behavior change and support patient self-management. J Clin Outcomes Manag 2014;21:17-29.

25. Tierney M, Fraser A, Purtill H, Kennedy N. Study to determine the criterion validity of the SenseWear Armband as a measure of physical activity in people with rheumatoid arthritis. Arthritis Care Res (Hoboken) 2013 Jun;65(6):888-895 [FREE Full text] [doi: 10.1002/acr.21914] [Medline: 23213019]

26. Holsgaard-Larsen A, Roos EM. Objectively measured physical activity in patients with end stage knee or hip osteoarthritis. Eur J Phys Rehabil Med 2012 Dec;48(4):577-585 [FREE Full text] [Medline: 22641252]

27. Semanik P, Lee J, Manheim L, Dipietro L, Dunlop D, Chang RW. Relationship between accelerometer-based measures of physical activity and the Yale Physical Activity Survey in adults with arthritis. Arthritis Care Res (Hoboken) 2011 Dec;63(12):1766-1772 [FREE Full text] [doi: 10.1002/acr.20644] [Medline: 21954166]

28. Almeida G, Wasko M, Jeong K, Moore C, Piva SR. Physical activity measured by the SenseWear Armband in women with rheumatoid arthritis. Phys Ther 2011 Sep;91(9):1367-1376 [FREE Full text] [doi: 10.2522/ptj.20100291] [Medline: 21719635]

29. Ainsworth B, Haskell EW. Compendium of Physical Activities Tracking Guide. URL: https://sites.google.com/site/ compendiumofphysicalactivities/ [accessed 2018-04-06] [WebCite Cache ID 6yU2kq5Q1]

30. Tremblay MS, Aubert S, Barnes JD, Saunders TJ, Carson V, Latimer-Cheung AE, SBRN Terminology Consensus Project Participants. Sedentary Behavior Research Network (SBRN) - Terminology Consensus Project process and outcome. Int J Behav Nutr Phys Act 2017 Dec 10;14(1):75 [FREE Full text] [doi: 10.1186/s12966-017-0525-8] [Medline: 28599680] 
31. Roos E, Roos P, Lohmander L, Ekdahl C, Beynnon BD. Knee Injury and Osteoarthritis Outcome Score (KOOS)--development of a self-administered outcome measure. J Orthop Sports Phys Ther 1998 Aug;28(2):88-96. [doi: 10.2519/jospt.1998.28.2.88] [Medline: $\underline{9699158]}$

32. Roos E, Roos P, Ekdahl C, Lohmander LS. Knee injury and Osteoarthritis Outcome Score (KOOS)--validation of a Swedish version. Scand J Med Sci Sports 1998 Dec;8(6):439-448. [Medline: 9863983]

33. Petkov J, Harvey P, Battersby M. The internal consistency and construct validity of the partners in health scale: validation of a patient rated chronic condition self-management measure. Qual Life Res 2010 Sep;19(7):1079-1085. [doi: 10.1007/s11136-010-9661-1] [Medline: 20437206]

34. Feehan L, Goldsmith CH, Leung AF, Li LC. SenseWearMini and Actigraph GT3X accelerometer classification of observed sedentary and light-intensity physical activities in a laboratory setting. Physiother Can 2016 May;68(2):116-123. [doi: 10.3138/PTC.2015-12]

35. Owen N. Sedentary behavior: understanding and influencing adults' prolonged sitting time. Prev Med 2012 Dec;55(6):535-539. [doi: 10.1016/j.ypmed.2012.08.024] [Medline: 22968124]

36. Dunstan D, Kingwell B, Larsen R, Healy G, Cerin E, Hamilton M, et al. Breaking up prolonged sitting reduces postprandial glucose and insulin responses. Diabetes Care 2012 May;35(5):976-983 [FREE Full text] [doi: 10.2337/dc11-1931] [Medline: 22374636]

37. Latouche C, Jowett J, Carey A, Bertovic D, Owen N, Dunstan D, et al. Effects of breaking up prolonged sitting on skeletal muscle gene expression. J Appl Physiol (1985) 2013 Feb 15;114(4):453-460 [FREE Full text] [doi: 10.1152/japplphysiol.00978.2012] [Medline: 23271697]

38. Howard B, Fraser S, Sethi P, Cerin E, Hamilton M, Owen N, et al. Impact on hemostatic parameters of interrupting sitting with intermittent activity. Med Sci Sports Exerc 2013 Jul;45(7):1285-1291. [doi: 10.1249/MSS.0b013e318285f57e] [Medline: 23439415]

39. Ory M, Resnick B, Jordan P, Coday M, Riebe D, Ewing GC, et al. Screening, safety, and adverse events in physical activity interventions: collaborative experiences from the behavior change consortium. Ann Behav Med 2005 Apr;29 Suppl:20-28. [doi: 10.1207/s15324796abm2902s_5] [Medline: 15921486]

40. Schoenfeld D. Statistical considerations for pilot studies. Int J Radiat Oncol Biol Phys 1980 Mar;6(3):371-374. [Medline: 7390914]

41. Carter R, Woolson RF. Statistical design considerations for pilot studies transitioning therapies from the bench to the bedside. J Transl Med 2004 Oct 28;2(1):37 [FREE Full text] [doi: 10.1186/1479-5876-2-37] [Medline: 15511289]

42. Lyons J, Lewis H, Mayrsohn G, Rowland JL. Behavior change techniques implemented in electronic lifestyle activity monitors: a systematic content analysis. J Med Internet Res 2014 Aug 15;16(8):e192 [FREE Full text] [doi: 10.2196/jmir.3469] [Medline: 25131661]

43. Task Force on Community Preventive Services. Recommendations to increase physical activity in communities. Am J Prev Med 2002 May;22(4 Suppl):67-72. [Medline: 11985935]

44. Lewis Z, Lyons E, Jarvis J, Baillargeon J. Using an electronic activity monitor system as an intervention modality: A systematic review. BMC Public Health 2015 Jun 24;15:585 [FREE Full text] [doi: 10.1186/s12889-015-1947-3] [Medline: 26104189]

45. Broekhuizen K, de Gelder J, Wijsman C, Wijsman L, Westendorp R, Verhagen E, et al. An Internet-based physical activity intervention to improve quality of life of inactive older adults: a randomized controlled trial. J Med Internet Res 2016 Apr 27;18(4):e74 [FREE Full text] [doi: 10.2196/jmir.4335] [Medline: 27122359]

46. Tulloch H, Fortier M, Hogg W. Physical activity counseling in primary care: who has and who should be counseling? Patient Educ Couns 2006 Dec;64(1-3):6-20. [doi: 10.1016/j.pec.2005.10.010] [Medline: 16472959]

47. Hutchison A, Breckon J, Johnston LH. Physical activity behavior change interventions based on the transtheoretical model: a systematic review. Health Educ Behav 2009 Oct;36(5):829-845. [doi: 10.1177/1090198108318491] [Medline: 18607007]

48. O'Donoghue G, Perchoux C, Mensah K, Lakerveld J, van der Ploeg H, Bernaards C, DEDIPAC Consortium. A systematic review of correlates of sedentary behaviour in adults aged 18-65 years: a socio-ecological approach. BMC Public Health 2016 Feb 17;16:163 [FREE Full text] [doi: 10.1186/s12889-016-2841-3] [Medline: 26887323]

49. Rutten G, Savelberg H, Biddle S, Kremers SP. Interrupting long periods of sitting: good STUFF. Int J Behav Nutr Phys Act 2013 Jan 02;10:1 [FREE Full text] [doi: 10.1186/1479-5868-10-1] [Medline: 23281722]

50. Gardner B, Smith L, Lorencatto F, Hamer M, Biddle SJ. How to reduce sitting time? A review of behaviour change strategies used in sedentary behaviour reduction interventions among adults. Health Psychol Rev 2016;10(1):89-112 [FREE Full text] [doi: 10.1080/17437199.2015.1082146] [Medline: 26315814]

51. Gardner B, Lally P, Wardle J. Making health habitual: the psychology of 'habit-formation' and general practice. Br J Gen Pract 2012 Dec;62(605):664-666 [FREE Full text] [doi: 10.3399/bjgp12X659466] [Medline: 23211256]

52. Gupta A, Tong X, Shaw C, Li L, Feehan L. In: Stephanidis C, editor. HCI International 19th International Conference, Proceedings, Part II. Cham, Switzerland: Springer; 2017:232-240.

53. Li LC, Feehan LM, Shaw C, Xie H, Sayre EC, Aviña-Zubeita A, et al. A technology-enabled counselling program versus a delayed treatment control to support physical activity participation in people with inflammatory arthritis: study protocol for the OPAM-IA randomized controlled trial. BMC Rheumatol 2017 Nov 28;1(1):1. [doi: 10.1186/s41927-017-0005-4] 


\section{Abbreviations}

BMI: body mass index

ICC: intraclass correlation coefficient

KOOS: Knee Injury and Osteoarthritis Outcome Score

MET: metabolic equivalent

MVPA: moderate-to-vigorous physical activity

OA: osteoarthritis

PAR-Q: Physical Activity Readiness Questionnaire

PT: physical therapist

RCT: randomized controlled trial

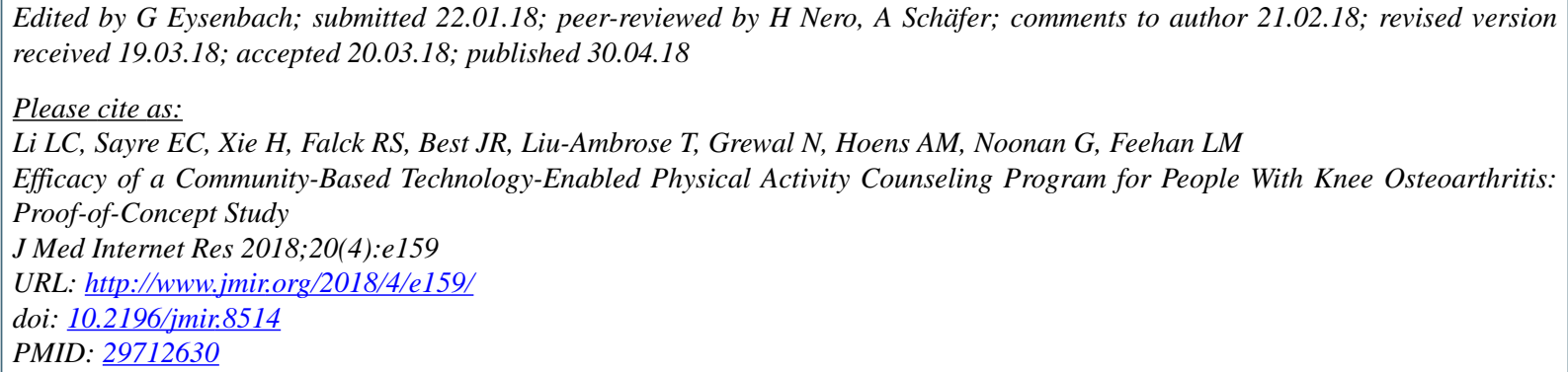

(CLinda C Li, Eric C Sayre, Hui Xie, Ryan S Falck, John R Best, Teresa Liu-Ambrose, Navi Grewal, Alison M Hoens, Greg Noonan, Lynne M Feehan. Originally published in the Journal of Medical Internet Research (http://www.jmir.org), 30.04.2018. This is an open-access article distributed under the terms of the Creative Commons Attribution License (https://creativecommons.org/licenses/by/4.0/), which permits unrestricted use, distribution, and reproduction in any medium, provided the original work, first published in the Journal of Medical Internet Research, is properly cited. The complete bibliographic information, a link to the original publication on http://www.jmir.org/, as well as this copyright and license information must be included. 